\title{
Period Changes of the Algol System SZ Herculis
}

\author{
J. W. Lee, C.-U. Lee, S.-L. Kim, H.-I. Kim, J.-H. Park and \\ T. C. Hinse
}

Korea Astronomy and Space Science Institute, Daejeon 305-348, Korea

email: jwlee@kasi.re.kr

\begin{abstract}
New CCD photometric observations of SZ Her were obtained between February and May 2008. More than 1,100 times of minimum light spanning more than one century were used for the period analysis. We find that the orbital period of SZ Her has varied due to a combination of two periodic variations, with cycle lengths of $P_{3}=85.8 \mathrm{yr}$ and $P_{4}=42.5 \mathrm{yr}$ and semi-amplitudes of $K_{3}=0.013$ days and $K_{4}=0.007$ days, respectively. The most reasonable explanation for them is a pair of light-time-travel (LTT) effects driven by the existence of two M-type companions with minimum masses of $M_{3}=0.22 M_{\odot}$ and $M_{4}=0.19 M_{\odot}$, located at nearly 2:1 mean motion resonance. Then, SZ Her is a quadruple system and the 3rd and 4th components would stay in the stable orbital resonance.
\end{abstract}

Keywords. binaries: close, binaries: eclipsing, stars: individual (SZ Herculis)

\section{Introduction}

Although the orbital period of SZ Her has been examined several times, its detailed study was made by Szekely (2003) and Soydugan (2008). The former reported that the period change can be fitted to a sine curve with a period of $66 \mathrm{yr}$, while the latter concluded that it can be represented by a single light-time-travel (LTT) ephemeris, caused mainly by a third body with a cycle length of $72 \mathrm{yr}$ and a minimum mass of $0.25 M_{\odot}$. Soydugan (2008) also suggested that the timing residuals from the LTT fit indicate an additional short-term oscillation with a period within about $20 \mathrm{yr}$. Nonetheless, the period variation still has not been studied as conclusively as can be desired. Here, we present that SZ Her is probably a quadruple system.

\section{Observation and result}

We performed new CCD photometry of SZ Her on 13 nights from 2008 February 28 through May 17. The observations were taken with a SITe $2 \mathrm{~K}$ CCD camera and a $B V R I$ filter set attached to the 61-cm reflector at Sobaeksan Optical Astronomy Observatory (SOAO) in Korea. The instrument and reduction method are the same as those described by Lee et al. (2007). GSC 2610-1116 and GSC 2610-0821, imaged on the chip at the same time as the program target, were selected as comparison and check stars, respectively. In addition to these, we observed two eclipse timing on both 2004 June and 2011 May, using $B$ and $B V$ filters attached to the $61-\mathrm{cm}$ reflector at SOAO, respectively.

Seventy-nine times of minimum light were determined from our observations and the WASP (Wide Angle Search for Planets) archive (Butters et al. 2010). For ephemeris computations, we have collected a total of 1050 timings from the literature to add to our measurements. First of all, we examined whether the period variations of SZ Her could be represented by the single-LTT effect as previous researchers have suggested. 
Table 1. Parameters for the LTT orbits of SZ Her.

\begin{tabular}{lccc}
\hline Parameter & $\tau_{3}$ & $\tau_{4}$ & Unit \\
\hline$T_{0}$ & \multicolumn{2}{c}{$2,434,987.39933(79)$} & HJD \\
$P$ & $0.818095788(46)$ & days \\
$a_{12} \sin i_{3,4}$ & $2.31(18)$ & $1.24(20)$ & AU \\
$\omega$ & $88.8(7.5)$ & $286(11)$ & deg \\
$e$ & $0.722(90)$ & $0.41(20)$ & \\
$n$ & $0.01149(13)$ & $0.02320(17)$ & deg day ${ }^{-1}$ \\
$T$ & $2,422,642(310)$ & $2,406,168(321)$ & HJD \\
$P_{3,4}$ & $85.8(1.0)$ & $42.5(1.1)$ & yr \\
$K$ & $0.0134(10)$ & $0.0071(11)$ & days \\
$f\left(M_{3,4}\right)$ & $0.00168(13)$ & $0.00106(17)$ & $\mathrm{M}_{\odot}$ \\
$M_{3,4} \sin i_{3,4}$ & $0.221(10)$ & $0.188(15)$ & $\mathrm{M}_{\odot}$ \\
\hline
\end{tabular}

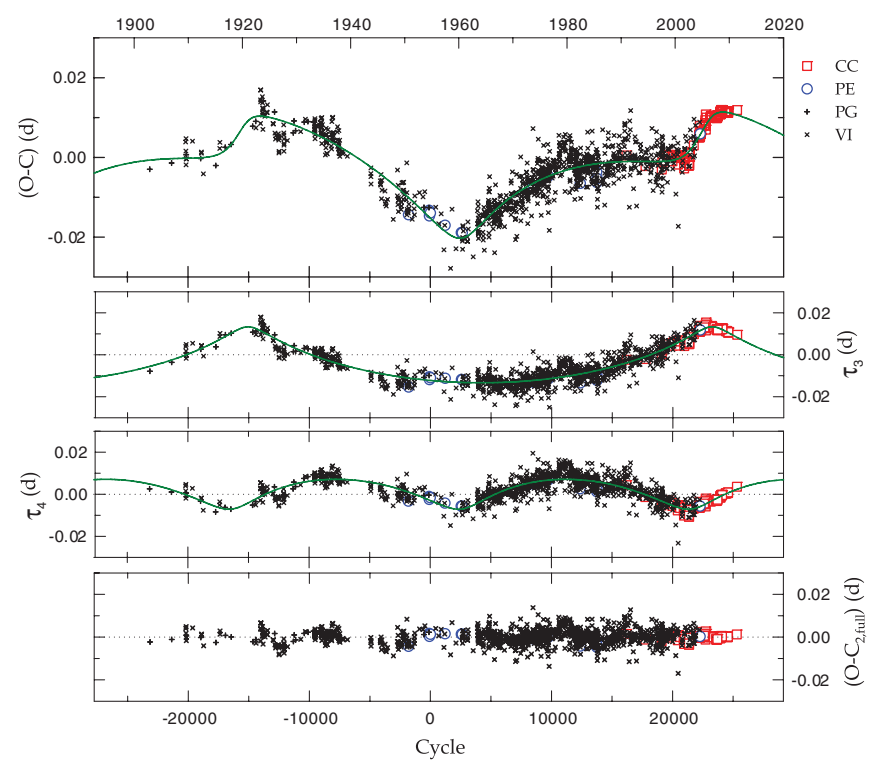

Figure 1. $O-C$ diagram of SZ Her. In the top panel, the continuous curve represents the full contribution of the ephemeris. The second and third panels display the long- and short-term LTT orbits, respectively, and the bottom panel the residuals from the complete ephemeris.

Fitting all timing residuals to that ephemeris form failed to give a satisfactory result. After testing several other forms, we found that the $O-C$ variation is best fitted by the two-LTT ephemeris, $C=T_{0}+P E+\tau_{3}+\tau_{4}$. Final results are summarized in Table 1 and plotted in Figure 1.

Because the LTT periods suggest nearly 2:1 resonant capture, the 3rd and 4th components would stay in the stable orbital resonance with a period ratio of $2.02 \pm 0.06$. If the two objects are on the main sequence and in the orbital plane $\left(i=87^{\circ} .6\right)$ of the eclipsing pair, the masses of the $3 \mathrm{rd}$ and 4 th bodies become $M_{3}=0.22 M_{\odot}$ and $M_{4}=$ $0.19 M_{\odot}$, respectively. Following the empirical relations, their radii and temperatures are calculated to be $R_{3}=0.23 R_{\odot}$ and $T_{3}=3017 \mathrm{~K}, R_{4}=0.20 R_{\odot}$ and $T_{4}=3008 \mathrm{~K}$.

\section{References}

Butters, O. W., West, R. G., Anderson, D. R., et al., 2010, A\& A, 520, L10

Lee, J. W., Kim, C.-H., \& Koch, R. H. 2007, MNRAS, 379, 1665

Soydugan, F. 2008, AN, 329, 587

Szekely, P. 2003, IBVS, 5467, 1 